\title{
La citología vaginal y la citologia del sedimento uterino en el embarazo ${ }^{(*)}$
}

Doctores Alfoso Alvarez Bravo, Mario González Ramos, Enrique Gutiérrez Murillo y Manuel Dosal. México, D. F.

A partir del descubrimiento del ciclo estral vagina! de los roedores debido a Stockard y Papanicolau (37) y de la descripción del ciclo vaginal humano hecha por Eliseo Ramírez en 1922 (29) y en 1928 (30), se perfiló un método de grandes alcances: la colpocitología, que quedó francamente establecido gracias a los estudios de Papanicolau y Papanicolau y Traut publicados en 1925 (23), 1933 (24) y 1943 (25).

El método desarrolló sus grandes posibilidades en manos de numerosos investigadores y en la actualidad es ampliamente usado tanto en su aspecto de citología funcional como para el diagnóstico del cáncer ginecológico. Fue este último aspecto, el citodiagnóstico del cáncer, perfeccionado e impulsado por Papanicolau y Traut (25), el que popularizó y dio un lugar definitivo a la citología vaginal en el arsenal del ginecólogo.

La aplicación del método a la obstetricia fue menos entusiasta, no obstante sus amplias posibilidades. Ya Papanicolau (23) en 1925 señaló en un breve artículo la importancia de la citología vaginal durante el embarazo, pero su utilidad pasó desapercibida.

Tinoco Cabral (38) en 1928, hizo una magnífica descripción del ciclo vaginal grávido-puerperal estudiado por el método colpocitológico. El mismo Papanicolau (24) en 1933 precisó aún más el cuadro citológico de la gravidez y Schuman (35) en 1944 publicó un trabajo en que señala la utilidad del método para el

(*) Ponencia presentada por la Asociación Mexicana de Ginecolıgía y Obstetricia en la VI Reunión Nacional de Ginecología y Obstetricia. León Gto., 9-13 de octubre de 1956. 
estudio de los trastornos del embarazo. La magnífica monografía de Pundel y Meensel (28) estudia los cuadros colpocitológicos en las distintas épocas del embarazo y señala, a semejanza de Hall (17) y de Benson y Traut (3), sil importancia pronóstica en la amenaza de aborto. Courrier (9) se muestra entusiasta partidario del método para el control clínico de los trastornos del embarazo, y Rodríguez Lima y Kamnitzer (32), en 1955, hacen un magnífico estudio de conjunto en el que reportan su experiencia tanto en el embarazo normal como en diversos trastornos de la gestación. Cohen y Rubinstein (8) y Bonime (5) han empleado el método para diagnóstico del embarazo, y Ezes (15) para el diagnóstico del embarazo postmaduro. Papanicolau (23) señaló ciertas modificaciones celulares que aparecen poco antes del parto. En los trabajos de Pundel y Meensel (28), Courrier (9) y otros se menciona también la existencia de modificaciones pre-partum del cuadro colpocitológico pero, sin embargo, no se les dió mayor importancia para el diagnóstico de la madurez del embarazo probablemente por la insuficiencia de los datos encontrados en los cuadros descritos. Como se verá más adelante, este aspecto de la citología funcional ha interesado nuevamente a últimas fechas y constituye ahora una de las aplicaciones importantes de la citología en obstetricia.

Del Castillo, Argonz y Galli Mainini (12), en 1946, basados en los estudios de Zuckerman $(39,40)$ y Cifuentes (7) acerca de la similitud embriológica y anatómica del epitelio vésico-uretral con el vaginal, demostraron que en las células descamadas presentes en el sedimento urinario se observan las mismas respuestas, tan ampliamente conocidas en citología vaginal, a la acción de las hormonas esteroides. Por ello idearon emplear, para la citología funcional, el estudio del sedimento urinario. Los estudios de Raynaud (31) y de Burns (6), acerca del origen y diferenciación de los epitelios y las modificaciones inducidas en ellos por los estrógenos, corroboran también este punto de vista.

Los mismos Del Castillo, Argonz y Galli Mainini $(13,14)$ demostraron posteriormente el paralelismo de las respuestas citológicas observadas en la citología vaginal comparada con la citología del sedimento urinario, durante el ciclo menstrual.

Lencioni (19) comprobó igualmente este paralelismo y calculó estadísticamente su correlación estableciendo entre la citología vaginal y la del sedimento urinario una media diferencia de $7.4 \% \pm 2.07$ (e. est.), derivada de la media general de células 
superficiales acidófilas de $24.9 \%$ encontrada en los frotis vaginales y la de $17.5 \%$ encontrada en los frotis del sedimento urinario: Asimismo estableció dos fórmulas reversibles para convertir los valores de la citología del sedimento urinario en los de la citología vaginal y viceversa, cuya correlación (r: 0.77) es altamente significativa.

El mismo Lencioni (20) y Pecorone, Belizan, Leoncioni y Berli (26) estudiaron también comparativamente el ciclo citológico vaginal y el del sedimento urinario durante el embarazo, encontrando una notable correlación entre ambos.

Mc Callin, Taylor y Whitehad (21) encontraron también variaciones significativas de la citología del sedimento urinario durante el ciclo menstrual y el ciclo grávido-puerperal y Rosen (33) reportó un extraordinario paralelismo entre las células descamadas observadas en los frotis vaginales y en los del sedimento urinario de recién nacidas, sobre todo en cuanto a su respuesta a la acción de los estrógenos.

Esta importante serie de investigaciones y nuestra propia experiencia, nos han llevado al convencimiento de que la citología del sedimento urinario empleada en su aspecto funcional, es decir, para el estudio de la acción de las hormonas esteroides, merece absoluta confianza y es del todo comparable a la citología vaginal.

La aparición de este nuevo método no tendría mayor importancia si sus ventajas se superpusieran a las de la citología vaginal, pero es evidente que la citología del sedimento urinario tiene las siguientes a su favor:

1. En los casos de infecciones e infestaciones vaginales los frotis vaginales son sucios y se presentan alteraciones celulares de ese origen, cosa que no sucede en los frotis del sedimento urinario.

2. Los frotis de sedimento urinario son incomparablemente más limpios y de más fácil lectura que los vaginales en los casos de pérdidas sanguíneas que acompañan a la gestación. Esto hace que deba preferirse cuando se emplea la citología para el control de la amenaza de aborto.

3. Para la toma del producto no es necesario que la enferma acuda al laboratorio, cosa inconveniente en los casos que se le ha prescrito reposo, ni tiene que recurrir a una persona entrenada en la toma del producto vaginal. Le basta con enviar una 
muestra de orina, después de varias horas de no haber orinado y previo aseo vulvar.

La confianza en el método de citología urinaria y las razones apuntadas, nos han llevado a preferirla sistemáticamente como medio de citología funcional, particularmente durante el embarazo.

\section{CITOLOGIA DEL CICLO GRAVIDO PUERPERAL}

Los estudios mencionados en las líneas anteriores y otros a que haremos referencia a continuación han llevado al conocimiento de los cuadros citológicos del ciclo grávido-puerperal, tanto vaginal como del sedimento urinario.

Las células vaginales, cervicales y vésico-uretrales descamadas durante el embarazo, salvo las particularidades a que se hará mención en su oportunidad, no se distinguen de las células encontradas fuera de la gravidez. A semejanza de lo que pasa en la citología funcional del ciclo menstrual, es la relación recíproca de los distintos elementos encontrados, lo que forma los diversos cuadros citológicos del ciclo grávido-puerperal. De alí la importancia de hacer colpocitogramas y urocitogramas por medio de la cuenta celular diferencial como lo han recomendado con grandes ventajas Ferin y Demol (16), De Allende y Orias (10), Pundel (27) y Schmitt (34).

El ciclo de descamación epitelia grávido-puerperal se caracteriza por la presencia de fenómenos proliferativos y de inhibición estrogénica, correlativos de la progresiva producción de las hormonas esteroides del ovario primero y de la placenta después, a los que sigue una fase regresiva inmediatamente anterior al parto, probablemente relacionada con fenómenos de senilidad placentaria, de acuerdo con los estudios de Smith y Smith (36).

En la primera semana del embarazo se aprecia el aspecto conocido en citología endócrina como "cuadro luteínico" pero con franca leucopenia. Predominan, pues, las células de la capa intermedia, basófilas, de bordes doblados, de núcleos vesiculosos o fusiformes, agrupadas frecuentemente en colgajos. Existę también las células "naviculares" de forma ojiva! y protoplasma limpio, descritas por Papanicolau (24).

Hasta la cuarta semana se conserva este cuadro con ligeras variaciones y se aprecia un aumento en el número de leucocitos. Los índices cariopicnótico y acidófilo no pasan del $5 \%$. 
De la quinta a la décima semana existen variaciones evidentes caracterizadas por un aumento progresivo de la acidofilia y la cariopicnosis que según Pundel y Meensel (28), Mussi y Falcoff (22) y Pecorone y colaboradores (26), llega hasta el 10 a $20 \%$, pero que según Rodríguez Lima y Kamnitzer (32) podría alcanzar valores hasta del 60 a $80 \%$ al final de este período. En citología urinaria los valores son inferiores. En nuestra experiencia nunca hemos encontrado indices superiores al $1.0 \%$. Este fenómeno de cornificación no ha sido explicado satisfactoriamente. Quizá sea debido, como se ha supuesto (28), al traspaso de la función de secreción esteroide, del ovario a la placenta. Durante este período vuelve, además, la leucopenia, desaparecen las células naviculares y hay ausencia, a veces completa, del fenómeno de aglutinación celular.

Del cuarto al sexto mes $(32,21)$, se establece el cuadro típico de embarazo, con reaparición de celdillas naviculares y leucocitos, disminución de los índices de cariopicnosis y acidofilia, incremento del fenómeno de aglutinación celular y del número de celdillas descamadas y notable reducción del número de celdillas de la capa superficial. Al cuarto mes el índice acidofílico es inferior al $10 \%$ y después baja al $5 \%$, para ser inferior al $2 \%$ hacia el noveno mes. Hacia el séptimo mes, según Papanicolau (24), las celdillas de la capa intermedia plegadas a nivel de sus bordes, adquieren la forma de "valvas de ostra".

El cuadro tipico de embarazo, que es la expresión de una actividad esteroide plena, ha sido descrito por dos de nosotros (1), en valores porcentuales de urocitograma, de la siguiente manera: Presencia de menos del $2 \%$ de celdillas acidófilas, menos del $10 \%$ de celdillas cariopicnóticas, menos del $20 \%$ de celdillas superficiales, abundancia de celdillas de la capa intermedia y presencia de celdillas naviculares y profundas.

Al final de la gestación desaparece el cuadro gravídico $\mathrm{y}$, aproximadamente una semana antes del parto, se presentan modificaciones sugerentes de la madurez del embarazo.

Los estudios hechos a propósito de esta última semana del embarazo han sido contradictorios y sus resultados no han sido definitivos porque han sido parciales y porque las modificaciones celulares se suceden con rapidez a través de estos últimos días del embarazo.

Ya dijimos que Papanicolau (23) señaló modificaciones celulares precursoras del parto. Pundel y Meensel (28) se refirie- 
ron al aumento del índice acidofílico, y de Wateville (11) en 1955 llamó la atención sobre el fenómeno de desvitalización celular. También en 1955 ratificaron parcialmente estos hallazgos Lemberg-Siegfied y Stamm (18) y Rodríguez Lima y Kamnitzer (38) y, en el presente año, Barnes y Zuspan (2).

Nuestros estudios, hechos en 1955 y reportados en la Primera Reunión Tocoginecológica Internacional de Cuba (1), basados en el Urocitograma, permitieron describir dos cuadros en los que se toman en cuenta los äiversos tipos celulares y su relación porcentual.

Estos cuadros fueron expresados en la siguiente forma:

a) Cuadros intermedios entre el patrón típico de embarazo y el de madurez gravídica, que aparecen de 3 a 7 días antes del parto y que se consideran como iniciación del proceso de senilidad placentaria: Presencia del 2 al 10\% de celdillas acidófilas, hasta el $30 \%$ de celdillas cariopicnóticas y hasta el $40 \%$ de celdilas superficiales, con disminución proporcional de las celdillas intermedias, naviculares y profundas.

b) Cuadro de madurez gravídica o de senilidad placentaria, oue se presenta dentro de los 3 días anteriores al parto: Presencia de más del $10 \%$ de celdillas acidófilas, más del $30 \%$ de celdillas cariopicnóticas y más del $40 \%$ de celdillas superficiales, disminución de las celdillas intermediarias y muy escasas o ausentes celdillas naviculares y profundas.

En la actualidad, como se verá en el curso de este trabajo, hemos tomado además en cuenta la presencia de leucocitos, de fenómenos de aglutinación y de desvitalización celular y de alteraciones nucleares. Además hemos dado importancia al hecho inusitado de que la eosinofilia predomina en las células intermediarias.

En el puerperio el frotis es de tipo atrófico, con muy escasas celdil'as superficiales y predominio de celdillas intermediarias y, sobre todo, de profundas.

Dado que el paralelismo indudable entre la citología vaginal y la del sedimento urinario tienen su correlación a distintos niveles (Lencioni) (19) hemos preparado el siguiente cuadro que da idea de los valores comparativos del índice acidófilico, de acuerdo con los trabajos de Pecorone y colaboradores (26). 


\section{CUADRO I}

VARIACION PORCENTUAL DE LA ACIDOFILIA DEL FROTIS VAGINAL Y DEL FROTIS DEL SEDIMENTO URINARIO EN EL EMBARAZO NORMAL

$\begin{array}{ccc}\begin{array}{c}\text { Mes del } \\ \text { embarazo }\end{array} & \begin{array}{c}\text { Colpocitograma } \\ \text { (Pr medio) } \%\end{array} & \begin{array}{c}\text { Urocitograma } \\ \text { (Promedio) \% }\end{array} \\ \text { I } & 4.60 & 3.10 \\ \text { II } & 10.76 & 4.73 \\ \text { III } & 9.08 & 5.11 \\ \text { IV } & 6.37 & 3 \\ \text { V } & 3.83 & 3.63 \\ \text { VI } & 1.84 & 2.70 \\ \text { VII } & 2.60 & 2.55 \\ \text { VIII } & 1.28 & 1.93 \\ \text { IX } & 1.63 & 0.63 \\ \text { 40a. Sem. } & 20.0 & 15.0 \\ \end{array}$

Tomado de Pecorone y Colab. (26), adicionado por nosotros con datos para el I mes y la última Semana del embarazo.

Puede apreciarse en este cuadro que la dispersión de valores de la acidofilia en la primera mitad del embarazo es mayor para la citología vaginal que para la del sedimento urinario, la cual puede ser debido, quizá a condiciones locales de la vagina, como piensan los autores de este estudio.

\section{MATERIAL Y METODO}

Por las ventajas que para estos estudios hemos reconocido a la citología del sedimento urinario, la hemos preferido y utilizado exclusivamente en el curso del presente trabajo.

Se siguió la técnica descrita por dos de nosotros (1) con modificaciones sugeridas posteriormente por González Ramos, que permiten obtener mayor riqueza celular y eliminan las alteraciones cromáticas celulares debidas a la desecación.

Preparación del frotis. 10 Se utilizó casi siempre la orina de la primera micción matutina, previo aseo vulvar. En algunas ocasiones se empleó la orina de una micción indeterminada, después de más de 3 horas de no haber orinado. 
$2^{\circ}$ Se centrifugaron dos porciones de 15 c. c. de orina durante 3 minutos a 1.000 revoluciones por minuto. En los casos en que el sedimento fue escaso, se repitió el proceso una o dos veces más.

$30^{\circ}$ Se decantó la orina y manteniendo el tubo invertido, se aspiró el sedimento adherido al tubo por medio de una pipeta de Pasteur. Se extendió suavemente el sedimento en dos portaobjetos que se sumergieron inmediatamente en una mezcla de partes iguales de alcohol-éter. González Ramos insiste en el hecho de mantener el tubo invertido durante la aspiración del sedimento con objeto de que no escurra orina de las paredes hacia el fondo, lo cual diluye el sedimento y altera su fijación.

Coloración.-El frotis puede teñirse con cualquiera de los métodos aconsejados para este tipo de estudios. Nosotros empleamos el método de coloración que hemos descrito en un trabajo previo (1) y el método clásico de Papanicolau.

Lectura.-Convencidos, como dijimos ya previamente, de las ventajas que para la interpretación de los frotis tiene el hacer la cuenta celular diferencial aconsejada por diversos autores (10, 16, 27, 34), no nos conformamos con apreciar el aspecto general del frotis y los caracteres celulares, sino que se hizo siempre un urocitograma diferencial, contando cuando menos 300 células de campos distintos, para expresar los valores porcentuales de celdillas acidófilas, de celdillas cariopicnóticas y de celdillas superficiales. Además se anotaron los siguientes caracteres: "Presencia de celdillas naviculares, características de las celdillas basófilas, existencia del fenómeno de aglutinación celuluar, presencia de alteraciones nucleares, existencia del fenómeno de aglutinación celular y presencia de leucocitos.

Material.—Se estudiaron 3.258 frotis de sedimento urinario correspondientes a 1.198 enfermas, 871 con embarazo normal y 327 con embarazo patológico.

Se empleó el método con diferentes objetos que aparecen enlistados en el cuadro II, en el cual se señala también el número de enfermas estudiadas y el número de estudios citológicos practicados: 


\section{CUADRO II}

MATERIAL DE ESTUUDIO DEL PRESENTE TRABAJO

\begin{tabular}{|c|c|c|}
\hline Objeto del estudio & $\begin{array}{r}N^{o} \text { de } \\
\text { enfermas }\end{array}$ & $\begin{array}{l}\mathrm{N}^{0} \text { de } \\
\text { Urocitogramas }\end{array}$ \\
\hline $\begin{array}{l}\text { Para diagnóstico del embarazo ........ } \\
\text { Para determinar la madurez del emba- } \\
\quad \text { razo: }\end{array}$ & 100 & 100 \\
\hline Estudios en las primeras horas del parto & 100 & 100 \\
\hline $\begin{array}{l}\text { Estudios al final del embarazo } \ldots \ldots \ldots \\
\text { Estudios en serie durante las dos últi- }\end{array}$ & 665 & 885 \\
\hline $\begin{array}{l}\quad \text { mas semanas del embarazo } \ldots . . \ldots \\
\text { Para control clínico de la amenaza de }\end{array}$ & 6 & 37 \\
\hline aborto $\ldots \ldots \ldots \ldots \ldots$ & 185 & 1.569 \\
\hline Casos de aborto incompleto ......... & 42 & 89 \\
\hline $\begin{array}{l}\text { Para control clínico de la amenaza de } \\
\text { parto prematuro } \ldots \ldots \ldots \ldots \ldots \ldots\end{array}$ & 31 & 125 \\
\hline Casos de embarazo ectópico $\ldots \ldots \ldots \ldots$ & 6 & 19 \\
\hline Casos de Mola hidatiforme $\ldots \ldots \ldots \ldots$ & 3 & 15 \\
\hline Casos de hiperemesis gravidica ...... & 8 & 77 \\
\hline Casos de pre-eclampsia y eclampsia .... & 39 & 182 \\
\hline Obito fetal in útero $\ldots \ldots \ldots \ldots \ldots \ldots$ & 6 & 22 \\
\hline Casos de placenta previa $\ldots \ldots \ldots \ldots \ldots$ & 7 & 38 \\
\hline TOTAL $\ldots \ldots \ldots \ldots$ & 1.198 & 3.258 \\
\hline
\end{tabular}

Método.-El método de estudio y manejo de los casos correspondientes a estos diversos grupos fue el siguiente:

Diagnóstico del embarazo.-Se estudiaron 100 enfermas con ciagnóstico o presunción clínica de embarazo y amenorrea de 6 a 10 semanas. En cada caso sa hizo una citología del sedimento urinario y una reacción de Galli Mainini. El criterio para diagnosticar embarazo por medio de la citología urinaría fue el enunciado al principio de este trabajo en relación con el cuadro citológico de las primeras 10 semanas del embarazo.

Determinación de la madurez del embarazo-Con este objeto se tomaron tres lotes distintos de enfermas. Un primer grupo 
de 100 casos en que se tomó la orina dentro de las primeras 6 horas de parto espontáneo, a término (39 a 40 semanas de embarazo), cuando la dilatación cervical tenía 3 a 4 centímetros. Se seleccionaron únicamente casos con antecedentes de embarazo normal, con presentación de vértice y membranas ovulares integras en el momento de recoger la orina.

En un segundo grupo de 665 casos se hizo un urocitograma a las 38 semanas del embarazo. Cuando el frotis no mostró signos de madurez para el parto se repitió el estudio cada semana hasta que el parto fue inducido o se inició espontáneamente.

En el tercer grupo, de 6 casos, se hizo un estudio en serie por medio de citologías practicadas cada 1 a 3 días hasta la iniciación del parto espontáneo.

Control clínico de la amenaza de aborto.-Se estudiaron 185 casos de mujeres con embarazo de 6 a 16 semanas, en quienes se hizo diagnóstico clínico de amenaza de aborto. Cuando el diagnóstico de embarazo no era clínicamente evidente se comprobó por medio de reacciones de Friedman o de Galli Mainini. Para el manejo de las enfermas se procedió de acuerdo con la siguiente secuela:

1.-Reposo y antiespasmódicos hasta tener el reporte del primer urocitograma.

2.-Urocitograma diario hasta obtener cuadros de actividad esteroide normal o hasta haber ausencia total de síntomas durante siete días.

3.-Se administró progesterona en los casos en que la citología mostró deficiencia de esta hormona, en las siguientes dosis: en los casos de deficiencia severa, 150 miligramos diarios, en las deficiencias moderadas, 120 miligramos diarios. Cuando se reportó insuficiencia estrogénica se administraron 150 miligramos diarios de dietilestilbestrol. En los casos de defciencia mixta se administraron diariamente 120 miligramos de progesterona y 150 mligramos de dietilestilbestrol.

4. - Se suspendió el tratamiento cuando el cuadro citológico fue normal o cuando hubo ausencia total de síntomas durante 7 días.

5.-Siempre que fue posible se controló a las enfermas que continuaron su embarazo por medio de estudios citológicos semanarios durante 2 o 3 semanas. 
Aborto incompleto.-Para conocer el cuadro citológico urinario de esta entidad clínica se hicieron estudios en 42 enfermas. Fara conocer la respuesta del epitelio uretro-vesical a las hormonas esteroides administradas, se continuó el tratamiento de progesterona y dietilestilbestrol, a pesar de su inutilidad terapéutica, y se hicieron urocitogramas diarios hasta que se practicó el legrado del útero. En todos los casos, el producto del legrado mostró la existencia de restos coriales.

Parto prematuro.-Se hizo el estudio citológico del sedimento urinario diariamente hasta la cesación de las contracciones uterinas o hasta que se efectuó el parto prematuro.

Embarazo ectópico.-Se hizo urocitograma diario, durante dos o tres días en 6 enfermas con embarazo ectópico, en proceso cie aborto o de ruptura. Todas ellas fueron operadas y se comprobó el diagnóstico durante el acto quirúrgico.

Mola hidatiforme.-En 3 casos de embarazo molar comprobado se hizo el estudio citológico urinario diariamente hasta que se hizo el vaciamiento uterino.

Hiperemesis gravidica.--En este grupo se hizo un urocitograma diario hasta la desaparición del síndrome.

Pre-eclampsia y eclampsia.-En estas enfermas se hizo diariamente el estudio cito'ógico del sedimento urinario hasta que se controló clínicamente la gestosis o hasta que se interrumpió el embarazo.

Obito fetal in útero.-En 6 casos de muerte fetal in ütero, comprobados clínica y radiológicamente, se hicieron 2 a 4 estudios citológicos. La muerte fetal prenatal se comprobó, además, al nacimiento del producto.

Placenta previa.-Se hizo un estudio cito ógico diario en las enfermas de este grupo hasta la suspensión del sangrado o hasta que fueron intervenidas quirúrgica $u$ obstétricamente.

\section{RESULTADOS}

Presentamos los resultados obtenidos de acuerdo con los grupos que fueron objeto del estudio.

Diagnóstico del embarazo.-En 80 casos se obtuvieron resultados positivos de embarazo tanto con el urocitograma como con la reacción de Galli Mainini. En 10 casos coincidió también la 
citología urinaria con la reacción biológica en sus resultados negativos. En 6 casos de R. de Galli Mainini positiva, el urocitograma no mostró el cuadro citológico de embarzo y en 4 casos en que la reacción biológica fue negativa, se hizo diagnóstico citológico positivo (cuadro III). En resumen, los resultados de la citología del sedimento urinario en comparación con la reacción de Galli Mainini, le conceden una exactitud de $90 \%$.

\section{CUADRO III}

ESTUDIO COMPARATIVO DE LA CITOLOGIA DEL SEDIMENTO URINARIO Y DE LA REACCION DE GALLI MAININI, PARA EL DIAGNOSTICO DEL EMBARAZO (100 CASOS)

Concordancia:

Citología y R. de Galli Mainini positivas ....... 80

Citologia y R. de Galli Mainini negativas ....... 10

TOTAL $\ldots \ldots \ldots \ldots \ldots \ldots, 90$

Discordancia:

Citología negativa y R. de Galli Mainini positiva 6 Citología positiva y $R$. de Galli Mainini negativa 4

TOTAL $\ldots \ldots \ldots \ldots \ldots \ldots \ldots, 10$

Determinación de la madurez del embarazo.-Los resultacios obtenidos en los tres grupos estudiados fueron como sigue:

En 99 de las 100 enfermas en quienes se practicó el estudio citológico urinario en las primeras horas del parto espontáneo a término, se apreció un cuadro citológico típico, no comparable a ninguna otra condición endocrinológica o gravídica. En un caso el cuadro fue incompleto según se verá después.

El cuadro típico de referencia puede describirse así:

1.--Relación porcentual de células:

Celdillas acidóficas:

De 13 a $62 \%$ (promedio: $22.3 \%$ )

Celdillas cariopicnóticas:

De 16 a $36 \%$ (promedio: $19.9 \%$ )

Celdillas superficiales:

De 12 a $47 \%$ (promedio: $22.7 \%$ )

Celdillas basales:

De 3 a $19 \%$ (promedio: 9\% ) 
2--Fenómeno de aglutinación celular: Este fenómeno se apreció solo en el 4\% de los casos y en ellos las agrupaciones celulares fueron poco numerosas.

3.-Fenómenos de desvitalización celular: En el $96 \%$ de los casos se encontraron alteraciones celuares caracterizadas por disminución notable del apetito tintorial, citoplasma irregular. contornos celulares esfumados y, con frecuencia, citolisios avanzada.

4.-Alteraciones nucleares: En el $100 \%$ de los casos se encontraron alteraciones nucleares en las celdillas intermediarias y basaless. Estas consistieron en la presencia de núcleos grandes en el $50 \%$ de los casos y existencia de células multinucleadas (2 a 6 núcleos por célula) en el $66 \%$.

5.-Nos llamó la atención que buena parte ne las celdillas acidófilas son de las de tipo intermediario.

6.-Leucocitos.-En $76 \%$ de los casos no había leucocitos 0 eran escasos. En el $24 \%$ restante se encontraron en mediana abundancia.

Solo en un caso, como ya se dijo, el cuadro fue incompleto por tener un indice acidófilo de $4 \%$ y un índice cariopicnótico de $6 \%$. Había sin embargo, evidentes fenómenos de desvitalización celular y alto porcentaje de alteraciones nuc'eares.

En resumen, el cuadro citológico urinario del principio de! parto que encontramos en el $99 \%$ de los casos se caracteriza por una disparidad entre una reacción acidófila intensa y una cariopicnosis discreta, por la presencia de buen número de celdillas basales profundas, sobre todo si se compara con el cuadro inmediatamente anterior al parto, por la ausencia de aglutinación celular, por la presencia de fenómenos muy constanteśs de desvitalización celular, por la presencia de francas alteraciones nucleares de las celdillas intermediarias y profundas, y por la poca cantidad de leucocitos.

En el grupo de casos estudiados semanariamente, en las últimas semanas del embarazo se encontraron los cuadros ya previamente descritos por dos de nosotros (1) y que han sido recordados al principio de este trabajo. Salvo en un caso que tenía un cuadro citológico inicial de maduración y que, sin embargo, dió a luz a las 24 horas, se corroboró que el patrón citơógico que hemos llamado "de madurez gravídica" aparece de 1 a 3 días 


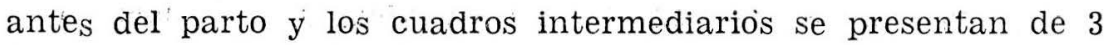
a 7 días antes del parto.

En el grupo de casos estudiados para este trabajo, hemos encontrado además de los datos de relación porcentual descritos por Alvarez Bravo y González Ramos (1) para el cuadro de "madurez gravídica", los siguientes hechos de interés.

1.-El fenómeno de aglutinación celular, que es típico del cuadro citológico del embarazo avanzado, es muy poco frecuen-te inmediatamente antes del parto $(10 \%)$.

2.-En el $40 \%$ de los casos existen fenómenos de desvitalización celular que excepcionalmente legan a los grados de citolisis encontrados por nosotros en los cuadros citológicos del principio del parto, pero que son francamente ostensibles.

3.-Existen las alteraciones nucleares que hemos descrito pa ra el cuadro citológico del principio del parto, aunque en menor proporción y menor intensidad.

4.-Hay leucopenia.

5.--Se aprecia el hecho inexplicable de que buena parte de las celdillas eosinófilas son de tipo intermediario.

En el grupo de casos en que se hizo una serie de estudios citológicos del sedimento urinario, cada 1 a 3 días, durante las dos últimas semanas del embarazo, se apreció el paso gradual del cuadro típico de embarazo, propio del 9o mes, a los cuadros intermediarios y por fin al de madurez para el parto. Se apreció en todos ellos que las modificaciones celulares son oscilantes y relativamente lentas (en 4 a 7 días) hasta llegar al cuadro de madurez que a veces entra en escena súbitamente, apenas unas horas o un día antes de la iniciación del parto.

Control clínico de la amenaza de aborto.-En 183 de los 184 casos estudiados por amenaza de aborto (99.4\%) se encontraron alteraciones del cuadro citológico propio del embarazo. El caso restante mostró un cuadro normal a pesar de existir pérdida sanguínea; se trató exclusivamente con reposo y cesó el sangrado en dos días, siendo dada de alta al 50 día en buenas condiciones.

Las alteraciones del cuadro citológico que fueron encontradas en los 183 casos fueron de tres tipos:

1.-En 149 casos, es decir en el $80.9 \%$ se apreció una elevación de los índices acidofílico y cariopicnótico por arriba de los valores normales, disminución o desaparición del fenómeno de 
aglutinación celular y de las celdillas naviculares. Este cuadro, que fue considerado como de insuficiencia progesterónica, se clasificó en cuatro grados: Una cruz $\left(^{+}\right)$: Indices acidofílico y cariopicnótico de 10 a $15 \%$ respectivamente.

Dos cruces $\left({ }^{++}\right)$: Indices acidofílico y cariopicnótico de 15 a $20 \%$. Persiste el fenómeno de aglutinación celular.

Tres cruces $(+++)$ : Indice acidofílico de 20 a $50 \%$ e indice cariopicnótico de 20 a $30 \%$; raramente se encuentran celdillas naviculares y aglutinadas.

Cuatro cruces $(++++)$ : Indice acidofílico de 20 a $50 \%$ e indice cariopicnótica de 30 a 50\%; no hay aglutinación celular ni celdillas naviculares; hay abundantes leucocitos.

2.-En 7 casos, o sea el $3.8 \%$, se encontró una disminución ostencible del fenómeno de proliferación de la capa intermedia, con la consiguiente disminución del número de celdillas de este tipo y aumento del número de celdillas basales. Se encontraron abundantes leucocitos. Los índices eosinofílico y cariopicnótico fueron normales. Este cuadro se consideró como de insuficiencia estrogénica.

3.-En 27 casos $(14.6 \%)$ se encontró un cuadro atrófico caracterizado por poca descamación celular, abundancia de celdillas basales, sobre todo profundas, con núcleos cromófilos, y abundantes leucocitos. Este cuadro se consideró como de insuficiencia mixta grave.

\section{CUADRO IV}

TIPOS DE ALTERACIONES CITOLOGICAS DEL SEDIMENTO URINARIO ENCONTRADAS EN 184 CASOS DE AMENAZA DE ABORTO

\begin{tabular}{lrr}
\hline & No & \multicolumn{1}{c}{$\%$} \\
\hline Cuadro normal de embarazo & 1 & 0.5 \\
Insuficiencia progesterónica & 149 & 80.9 \\
Insuficiencia estrogénica & 7 & 3.8 \\
Cuadro atrófico & 27 & 14.6 \\
\hline
\end{tabular}

Según se dijo en el capítulo precedente, en estas enfermas se controló la terapéutica por medio de citologías urinarias diarias. Las respuestas citológicas fueron irregulares, pues se estableció un sistema terapéutico rígido sin empeñarnos en obtener un cuadro citológico de actividad esteroide normal. Sin embar- 
go, en el $\mathbf{8 0 \%}$ de los casos se apreció una respuesta congruente con la terapéutica y con la evolución clínica de las enfermas. En 25 casos, en cambio, en que suspendió la terapéutica por ausencia total de síntomas durante 7 días, el urocitograma continuó mostrando insuficiencia progesterónica $+\mathrm{y}++$ a pesar de que el embarazo continuó normalmente cuando menos durante el período de 2 a 4 semanas en que estas enfermas fueron vigiladas. Asimismo, en 13 casos en que la citología de control terapéutico mostró actividad esteroide normal, se presentó el aborto.

Aborto incompleto.-En los 42 casos de aborto incompleto $(100 \%)$ se encontró un cuadro citológico de tipo atrófico caracterizado por abundancia de celdillas de la capa basal, sobre todo profundas, con núcleo cromófilo, poca descamación celular y abundantes leucocitos.

Con objeto de comprobar hasta dónde llega la respuesta del urocitograma a la administración de estrógenos y progesterona, estas enfermas fueron tratadas con dietilestilbestrol y progesterona de acuerdo con el método que empleamos para los casos ce amenaza de aborto y se aprecia una evidente mod'ficación dei cuadro citológico que en algunos casos llegó a tomar los caracteres propios del embarazo normal, no obstante la ausencia de un huevo activo.

Amenaza de parto prematuro.-En las 31 enfermas con amenaza de parto prematuro $(100 \%)$ se encontraron los cuadros citológicos regresivos que hemos descrito como propios de la última semana del embarazo normal. En todos los casos que fueron controlados y en los que continuó el embarazo, el cuadro citológico readquirió los caracteres propios del embarazo.

Embarazo ectópico.-En los 6 casos de embarazo ectópico (100\%) apreciaron modificaciones citológicas evidentes. Dos casos $(33 \%)$ dieron el cuadro citológico que ya hemos descrito y $4(66 \%)$ presentaron el cuadro de insuficiencia progesterónica que hemos calificado con 4 cruces $(++++)$. La reacción de Friedman fue positiva en 5 de estos casos y negativa en un caso.

Mola hidatiforme.-En ninguno de los 15 frotis del sedimento urinario correspondientes a las 3 enfermas estudiadas se encontraron alteraciones citológicas del cuadro de embarazo.

Hiperemesis gravídica.-En las 8 enfermas de este grupo $(100 \%)$ se apreció una intensa reacción de cornificación con ín- 
dice acidofílico hasta del $50 \%$ e índice cariopicnótico hasta del $40 \%$. En todos los casos, también se apreció una mejoría o normalización del cuadro citológico cuando desapareció el síndrome. Dos enfermas fueron tratadas exclusivamente con dosis altas de progesterona y 6 con dieta, reposo, sugestión, sedantes y anticolinérgicos.

Pre-eclampsia y eclampsia.-En 31 de los 39 casos de este grupo $(79.4 \%)$, en los cuales están incluídos 3 casos de eclampsia; la citología del sedimento urinario no mostró alteración alguna. En los 8 casos restantes $(20.5 \%)$ hubo elevación moderada (4 a 12\%) de los índices acidofílico y cariopicnótico. Hay que tomar en cuenta, sin embargo, que todas ellas tenían embarazos de 38 a 40 semanas y que 3 de ellas dieron a luz de uno a tres días después.

Obito Fetal in útero.-En los 6 casos de muerte fetal in útero (100\%) se encontró el cuadro típico atrófico que ya hemos descrito.

Placenta previa.-En 40 urocitogramas practicados en 8 enfermas con placenta previa se encontró siempre e cuadro típico de embarazo $(100 \%)$.

\section{COMENTARIO}

Es evidente que la citología vaginal y la citologia del sedimento urinario expresan claramente las condiciones hormonales del embarazo en lo que hace a las hormonas esteroides y que no muestran alteraciones citológicas relacionadas con el tenor de la hormona gonadotrópica. A este último propósito basta recordar que no encontramos alteración alguna en casos de mola hidatiforme en los que la increción gonadotrópica es tan intensa.

Después del estudio que se reporta en este trabajo tenemos el convencimiento de que la citología del sedimento urinario es tan sensible como la vaginal a las variaciones hormonales dei embarazo y tiene sobre ella indudables ventajas que ya han sido mencionadas y que le dan un lugar de privilegio en citología funcional y más particularmente durante el embarazo. El único obstáculo de la citología urinaria es la preparación del frotis, que es difícil. Sin embargo, gracias a la técnica descrita, desarrollada por uno de nosotros (González Ramos), es fácil y seguro obtener frotis con gran riqueza celular y sin alteraciones cromáticas artificiales. 
La relación que hemos hecho de los cuadros cito ógicos encontrados y nuestra experiencia, ponen de manifiesto la necesidad de hacer "urocitogramas" en los que se aprecie de manera fácil y segura la relación porcentual de las células presentes, sobre todo para la expresión de los índices eosinofílico y cariopicnótico. La cuenta diferencial da, además, una idea de a intensidad del trastorno.

A propósito de la utilidad de la citología del sedimento urinario durante el embarazo se ofrecen interesantes comentarios.

Para el diagnóstico temprano del embarazo nos parece de poca utilidad ya que su sensibilidad frente a una reacción biológica fue solamente del $90 \%$. Esta cifra es semejante a la reportada por Bonime (5) y ligeramente superior a la señalada por Mc Callin y colaboradores (21).

Para la determinación de la madurez del embarazo nos parece un método de gran alcance y de utilidad práctica. Numerosos autores, desde Papanicolau hasta la fecha, han encontrado alteraciones citológicas precursoras del parto. Los datos que ahora reportamos nosotros ponen claramente de manifiesto la existencia de variaciones progresivas y muy particulares de la última semana del embarazo. Sin embargo, hay anarquía de resultados y poco entusiasmo de algunos autores porque sus estudios han enfocado sólo parcialmente el problema.

Así, por ejemplo, Pundel y Meensel (28) han estudiado so'amente el aumento del indice eosinofilico, de Watteville (11) ha basado únicamente sus trabajos en la presencia de celdillas desvitalizadas, Lemberg-Siegfried y Stamm (18) en el fenómeno de cornificación y Barnes y Zuspan (2) en la ausencia de ce'dillas naviculares, la presencia de celdillas desvitalizadas, la falta de apetito tintorial y la presencia de leucocitos y moco. Las cifras dadas por estos autores como indice de frecuencia de la aparición de los cuadros por ellos tomados en cuenta varía del 77 al $90 \%$. En estudios cronológicamente simultáneos o anteriores a los que acabamos de referir, dos de nosotros (1) encontraron por medio de la cuenta celular diferencial, datos evidentes para determinar la proximidad del parto y precisar la fecha de aparición de estos fenómenos. En el estudio presente se tomaron 100 orinas en las primeras horas del parto y se encontró en el $99 \%$ de los casos un cuadro tan típico y particular que lo hace inconfundible. De gran importancia en el reconocimiento de este cuadro, además de la cuenta celular diferencial, son la au- 
sencia de aglutinación celular, los fenómenos de desvitalización celular, la presencia de alteraciones nucleares y la existencia de numerosas celdillas intermediarias eosinofílicas. Si se toman en cuenta todos estos factores se apreciará que los cuadros citológicos inmediatamente previos al parto, ya intermediarios o de madurez gravídica, son semejantes al cuadro del principio del parto, pero con alteraciones menos marcadas, aunque igualmente reconocibles. En nuestra experiencia, reportada en este trabajo, es posible determinar la proximidad del parto, con una antelación de 1 a 7 días, en casi el $100 \%$ de los casos. De los signos encontrados en los frotis tienen particular valor para el diagnóstico de madurez, en orden de importancia semiológica, la elevación del índice acidofílico, la presencia de fenómenos de desvitalización celular, la elevación del índice cariopicnótico, la desaparición de los grupos de células aglutinadas, la presencia ae alteraciones nucleares y la existencia de celdillas intermediarias acidófilas. La presencia de estas últimas, que constituye un fenómeno inexplicable, ha sido reportada por Bernstein y Rakoff (4) en la amenaza de aborto.

Creemos que deben considerarse los cuatro cuadros citológicos siguientes:

I. - Cuadro de actividad esteroide plena, típico del $9^{\circ}$ mes del embarazo.

II.-Cuadros intermediarios que aparecen de 3 a 7 días antes del parto.

III.-Cuadro de madurez gravídica para el parto.

IV.-Cuadro citológico de principio del parto.

Estos cuatro cuadros han sido ya descritos en el curso de este trabajo. La interpretación de su causa es difícil, pues ni se trata simplemente de una reacción estrogénica de cornificación, ni es propiamente un cuadro regresivo como sugieren Rodríguez Lima y Kamnitzer (32). Al lado de estos fenómenos hay otros que no podemos explicar, como la acidofilia de las células intermediarias, la disparidad entre los índices acidofílico y cariopicnótico que se presenta en el momento del parto, la presencia de alteraciones nucleares (sobre todo la aparición de células multinucleadas), los cuales pudieran estar, quizá en relación con algún fenómeno desencadenante del parto. Pensamos en esta posibilidad basados en el hecho de que las alteraciones iniciales, que por cierto se establecen con relativa lentitud, consisten casi 
exclusivamente en la elevación de los índices de acidofilia y cariopicnosis, y tcdos los demás fenómenos aparecen bruscamente después, muy poco antes del parto, y se establecen francamerte en cuanto éste empieza.

Para el control clinico de la amenaza de aborto el urocitograma es un recurso auxiliar de valor. Según Pecorone y colaboradores (26), que hicieron un estudio comparativo, es superior a la citología vaginal por suprimir las causas de error derivadas de las condiciones locales de la vagina y dar índices más uniforines. El valor de la citología vaginal o urinaria para el diagnóstico de las condiciones hormonales del embarazo amenazado de aborto, es evidente según la estadística que reportamos y la experiencia de Bonime (5), Courrier (9), y Traut (3) y otros. El valor diagnóstico del urocitograma en nuestros casos fue de $99 \%$.

Las alteraciones encontradas consisten fundamentalmente en una elevación de los índices acidofílico y cariopicnótico. Interesa, además, el apreciar la deficiencia de proliferación de la capa intermedia propia de la insuficiencia estrogénica que, por prmera vez, se reporta en este trabajo.

Vale la pena insistir en que tanto para reconocer fácilmente las alteraciones celulares, cuanto, sobre todo, para tener una idea de su intensidad que permita evaluar la gravedad del trastorno, es indispensable hacer la cuenta diferencial de los índices.

En otras palabras, es indispensable hacer colpocitogramas o urocitogramas.

Para el control terapéutico es también de utilidad pero debe saberse interpretar, pue $e_{S}$ refleja las variaciones hormonales tanto ováricas o placentarias como terapéuticas, de manera que puede llegar a obtenerse un cuadro citológico de embarazo normal existiendo un huevo muerto, como sucedió en los casos que hemos reportado. Además, nuestra serie de 42 casos de aborto incompleto a los que se administraron altas dosis de estrógenos y progesterona confirma también este punto de vista. Por ello creemos que el método es útil para asegurar un nivel terapéutico adecuado de las hormonas administradas, pero no para dar una idea del estado del huevo ni del pronóstico del caso. Es indispensable, por tanto, recurrir a la exploración clínica y a la dosificación de gonadotropinas para evitar la prolongación torpe de una terapéutica inútil.

Dado su valor diagnóstico, el método es útil para el control del caso en las semanas siguientes a la supresión del tratamien- 
to, pues con frecuencia anuncia la recaída con gran oportuniciad. En ahorro de tiempo evitamos referir los casos evidentes de este tipo que tuvimos la oportunidad de observar en esta serie.

Por lo anteriormente dicho se comprende que el urocitograma en estos casos sólo es útil cuando se practica en serie, cada 1 a 3 o 7 días, según las variaciones encontradas y la evolución clínica.

En los casos de amenaza de parto prematuro y como era de esperarse, se obtienen cuadros citológicos semejantes a los que aparecen a término. Si se recuerda que según la experiencia obtenida en el estudio de nuestros 31 casos, los cuadros regresan cuando se estabiliza el embarazo, se comprenderá que, aclemás de la utilidad del urocitograma para confirmar la amenaza y diferenciarla del falso trabajo prematuro, permite por comparación de los cuadros sucesivos, tener un recurso pronóstico auxiiiar. Además, cuando aparece un cuadro citológico similar al que hemos descrito como de "madurez gravídica", revela la inminencia de la expulsión del producto.

En los casos de embarazo ectópico en proceso de aborto o de ruptura, la citología del sedimento urinario da cuadros atróficos reveladores de la muerte del huevo que pueden ser de gran utilidad para el diagnóstico diferencial con los procesos inflamatorios de la pelvis.

En nuestros casos de mola hidatiforme la citología urinaria no dio ningún dato de importancia que pudiera hacerla aplicable a la clínica de este padecimiento.

En cambio nos parece de gran interés la intensa reacción de cornificación apreciada constantemente en los frotis de sedimento urinario en los casos de hiperemesis gravidica, ya señalada previamente por Bonime (5), así como la regresión del fenómeno conforme mejora la paciente. Esto permite suponer que en el fondo de este trastorno existe una alteración hormonal causal o secundaria que sería muy interesante de estudiar tanto desde el punto de vista etiológico como desde el terapéutico.

Los resultados obtenidos en los 39 casos de preeclampsia y eclampsia no muestran tampoco ningún dato de interés. Los pocos casos en que se apreció una elevación del índice acidófilo estaban muy cerca del término, por lo que no pueden tomarse en cuenta ya que este signo bien pudo corresponder a los fenómenos prepartum que hemos encontrado como expresión de la madurez gravídica. Se ocurre, por tanto, que la senilidad placentaria in- 
vocada por Smith y Smith en las toxemias; no se confirma, al me. nos en lo que hace a la respuesta del efector epitelial uretro-vesical.

Nos parece de trascendental importancia diagnóstica por la constancia y precisión del cuadro citológico urinario, el valor de la citología en los casos de muerte fetal in útero. Este cuadro atrófico, cuyo valor diagnóstico ha sido particularmente enfatizado por Rodríguez Lima y Kamnitzer (32) en sus estudios de citología vaginal, tiene su traducción exacta en citología del sedimento urinario y adquiere un valor casi patognomónico. Ya dijimos que este cuadro aparece también en los casos de muerte ovular en la primera mitad del embarazo (aborto con huevo muerto o parcialmente expulsado, embarazo ectópico roto), casos en los que tiene la misma fuerza semiológica.

Finalmente, y como era de preverse, la citología del sedimento urinario no muestra alteraciones durante el sangrado de la placenta previa, ya que en los casos en que pudiera aplicarse el método, el desprendimiento placentario es parcial y de poca importancia frente a la actividad funcional de la placenta.

El análisis de los resultados reportados en este trabajo pone de relieve la sensibilidad de la citología del sedimento urinario para revelar las variaciones del nivel hormonal esteroide, tanto en el embarazo normal como en el patológico y pone de manifiesto su utilidad como precioso auxiliar de la clínica obstétrica.

\section{RESUMEN Y CONCLUSIONES}

1. Se reportan y analizan los resultados obtenidos por medio del estudio citológico de 3.258 frotis de sedimento urinario correspondientes a 1.198 pacientes con embarazo normal y patológico.

2.-No se encontraron alteraciones citológicas de importancia en los casos de mola hidatiforme, de preeclampsia y eclampsia y de placenta previa.

3.-Utilizando este método de citología para el diagnóstico temprano del embarazo en comparación con la reacción biológica de Galli Mainini, se encontró un coeficente de exactitud de solo $90 \%$.

4.-Se apreciaron típicas alteraciones del cuadro citológico del embarazo en la última semana de la gravidez y en el principio del parto, que permiten describir cuatro cuadros citológicos, a saber: 
a) Cuadro del embarazo avanzado normal que sugiere la existencia de una actividad esteroide plena.

b) Cuadros intermediarios, que aparecen de 3 a 7 días antes del parto espontáneo, caracterizados por una elevación moderada de los índices acidofílico y cariopicnótico.

c) Cuadro de madurez gravídica, que aparece de 1 a 3 días antes del parto, caracterizado por una acidofilia mayor (superior $10 \%$ ), aumento de la cariopicnosis (más del $30 \%$ ), ausencia de grupos de células aglutinadas, presencia de fenómenos de desvitalización celular, existencia de alteraciones nucleares (sobre todo aparición de células multinucleadas) y presencia de celdillas intermediarias eosinófilas.

d) Cuadro citológico del principio del parto, semejante al anterior pero con las siguientes peculiaridades que lo hacen típico: disparidad entre los índices acidofílico y cariopicnótico, ausencia de aglutinación celular, intensa citolisis y gran número de células con alteraciones nucleares.

5.-En los casos de muerte del huevo o de expulsión parcial del mismo (huevo muerto y retenido, aborto incompleto, embarazo ectópico en proceso de aborto o de rotura y óbito fetal in útero) se encontró en los frotis un cuadro atrófico, bien distante del propio del embarazo, en el $\mathbf{1 0 0 \%}$ de los casos, por lo que le concedemos gran valor diagnóstico.

6.-En los casos de amenaza de aborto se encontraron alteraciones reveladoras de insuficiencia progesterónica en el $80.9 \%$ de los casos, signos de insuficiencia estrogénica en el $3.8 \%$ y alteraciones que fueron interpretadas como de insuficiencia mixta en el $14.6 \%$. El valor dagnóstico y de orientación terapéutica de la citología urinaria en estos casos, nos parece indudable.

7.-En el control terapéutico de la amenaza de aborto la citologia del sedimento urinario es de utilidad para proporcionar las dosis a las necesidades del caso. Debido a que el urocitograma se modifica de acuerdo con las hormonas administradas terapéutimente, deja de tener valor para revelar las condiciones hormonales propias de la gestación desde el momento en que entran en circulación los esteroides exógenos. Una vez suspendido el tratamiento la citología urinaria es de gran utilidad para seguir la evolución del caso.

8.-En la amenaza de parto prematuro el urocitograma es útil para descartar los casos de falso trabajo de parto, para ad- 
vertir la inminencia del parto y para seguir la evolución del caso que tiende a ser controlado.

9.-En el $100 \%$ de los casos de hiperemesis gravídica se encontró intensa reacción de cornificacióón que hace suponer la existencia de un fondo hormonal de estos trastornos, que merece la pena estudiarse.

10. - La citología del sedimento urinario reveló una alta sensibilidad a las variaciones de la actividad esteroide placentaria, tanto en el embarazo normal como en el patológico.

11. - Se insiste en la conveniencia de hacer "urocitogramas" con cuenta diferencial de células, que permitan no solamente reconocer el trastorno, sino tener una idea de su intensidad.

\section{BIBLIOGRAFIA}

1. Alvarez BRAVo, A. y GONZALez RAMOS, M. - Ginec. y Obstet. Méx. 11: 231, 1956.

2. BARNES, A. C., and zUSPAN, F. P. $\rightarrow$ Am. J. Obst. \& Gyn. 71: 1080; 1956.

3. BENSON, R. C. y TRAUT, H. F. - J. Clin. Endocrinol., 10: 675, 1950.

4. BERNSTEIN, J. B. y RAKOFF, A. E. - Vaginal Infections, Infestations, and Discharges. The Blakiston Co. Inc. New York-Toronto, 1953, pág. $55-56$.

5. BONIME, R. G. - Am. J. Obst. \& Gyn. 58: 524; 1949.

6. BURNS, R. K. - Carnegie Inst. of Washington Publication, No $^{\circ} 541$ : $53 ; 1942$.

7. CIFUentes DELATTE, L. - Rev. Clin. Espñ., 20: 54. 1946.

8. COHEN, M. R. y RUBinstein, B. B. - Proc. Inst. Med. Chicag ?, 14: $369,1943$.

9. COURRIER. - En Pundel y Meensel. Loc. Cit.

10. DE ALLENDE, J. L. C. y ORIAS, O.: Cytology of the Human Vagina.

Paul B. Hoeber, New York, 1950.

11. De WATteville, H. - Memorias del 2o Congreso Mexicano de Ginecología y Obstetricia. Tomo II. México D. F., 1955.

12. DEL CASTILLO, E. B., ARGONZ, J. y GALLI MAININI, C. - Semana Médica, 53: 867, 1946.

13. DEL CASTILLO, E. B., ARGONZ, J. y GALLI MAININI, C. - J. Clin Endocrinol. 8: 76, 1948.

14. DeL CASTILLO, E. B., ARGONZ, J. y GALli MAININI, C. - J. Clin Endocrinoī. 9: 1362, 1949.

15. EZES, H. - Ann Endocrinol. 14: 463. 1953.

16. FERIN, J. y DEMOL, R. - Ann d'Endocrinologie, 11: 668, 1950.

17. Hall, G. J. - J. Clin. Endocrin., 5: 34, 1945. 
18. LEMEERG-SIEGFRIED and STAMM, O. - Gebrurtsh. uu. Frauenh. 15: 885-397, 1955.

19. LENCIONI, L. J. - J. Cl. End. and Mctab., 13: 263. 1953.

20. LENCIONI, L. J. - Semana Médica. 104: 346, 1954.

21. Mc CALLIN, P. F., TAYLOR E. S., WHITEHEAD, R. N. - Am. J. Obst. \& Gyn. 60: 64, 1950.

22. MUSSI, F. \& FALCOFF, F. - Obst. y Ginec. Latino-Am. 10: 449, 1952.

23. PAPANiCOlaU, G. N.-Proc. Soc, Exper. Biol. \& Med., 22: 436. 1925.

24. PAPANICOLAU, G. N. - Am. J. Anat., 52: 519; 1933.

25. PAPANICOLAU, G. N. y TRAUT, H. F. - Diagnosis of Uterine Cancer by the Vaginal Smear. The Commonwealth Fund. New York, 1943.

26. PECORONE, R. BELIZN, L. A., LENCIONI, L. J. y BERLI, R. - Ginec. Lat. Am., 13: 145; 1955.

27. PUNDEL, P. - Acta Clin Belgique, 5: 66, 1950.

28. PUNDEL, J. P. y MEENSEL, F. V. - Gestation et Cytologie Vaginale. La Clinique Universitaire Bruggmann. Monsonet Cie., 1951.

29. RAMIREZ, E. - Rev. Mex. de Biol. 2: 199; 1922.

30. RAMIREZ, E. - Rev. Mex. de Eiol. 8: 1; 1928.

31. RAYNAUD, A. - Compt rend. Soc. de Biol. 126: 215; 1937.

32. RODRIGUEZ LIMA y DE BENNING KAMNITZER, M. - Rev. de Obst. y Gin. Caracas. 15: 977; 1955.

33. ROSEN, M. I. - Am. J. Obst. \& Gynec. 65: 376; 1955.

34. SCHMITT, A. - Gebrurtsh. u. Frauenh. 13: 593; 1953.

35. SCHUMAN, W. - Am. J. Obst. Gin. 47: 808; 1944.

36. SMITH, G. V. S., y SMITH, O. W. - J. Clin. Endocrinol., 1: 470; 1941.

37. STOCKARD y PAPANICOALU, G. N. - Am. J. Anat., 22: 225. 1917.

38. TINOCO CABRAL. - Modificacoes das Células Epiteliais da Vagina Humana na Gravidez en na Menopausa. Tesis. San Paulo, 1928.

39. ZUCKERMAN, S. - Lancet 1: 135; 1938.

40. ZUCKERMAN, S. - Biol. Rev., 15: 231; 1940.

La dirección de la Revista, a todos sus suscriptores, de la manera más atenta, se sirvan enviar a la Direcciónl los siguientes números con el abjeto de atender solicitudes de canje extranjero que no podemos cumplir:

Volumen I - Todos.

Volumen II - Números 1, 3, 4, 5 .

Volumen III - Números 1, 5.

Volumen IV - Números 2, 3, 5, 6.

Volumen $V$ - Número 1. 